\title{
Chirality selection in the vortex state of magnetic nanodisks with a screw dislocation
}

\author{
A. B. Butenko ${ }^{1, a}$ and U. K. Rößler ${ }^{1}$ \\ IFW Dresden, Helmholtzstr. 20, 01069 Dresden, Germany
}

\begin{abstract}
Structural defects in magnetic crystalline materials may locally change magnetic properties and can significantly influence the behavior of magnetic nanostructures. E.g., surface-induced Dzyaloshinskii-Moriya interactions can strongly affect vortex structures in magnetic nanodisks causing a chirality selection. Near lattice defects, the spin-orbit interactions induce local antisymmetric Dzyaloshinskii-Moriya exchange and cause effective anisotropies, which can result in spin canting. Broken inversion symmetry near a defect leads to locally chiral exchange. We present a phenomenological approach for dislocation-induced Dzyaloshinskii-Moriya couplings. As an example we investigate effects of a screw dislocation at the center of a magnetic nanodisk with a vortex state. By numerical calculations on vortex profiles we analyze equilibrium parameters of the vortex as functions of applied magnetic field and the material and geometrical parameters. It is proposed that magnetic nanodisks with defects provide a suitable experimental setting to study induced chirality by spin-orbit effects.
\end{abstract}

\section{Introduction}

Defects break the local symmetry of the crystal structure and induce inhomogeneous magnetic couplings. Thus defects can act as a source of additional local interactions. Depending on remaining symmetries, the induced magnetic couplings may consist of (i) local anisotropies and (ii) chiral magnetic Dzyaloshinskii-Moriya (DM) couplings that can favour non-collinear magnetization structures [1] similar to the chiral modulated states in magnetic crystals from noncentrosymmetric crystal classes [2]. Because of the variety of different types and arrangements of defects and their interactions with one another and with magnetic textures, the effects of defects on magnetic behavior then are very complex [3] [4]. While planar defects have been frequently studied, the experimental investigations of individual line or point defects were hindered for a long time by spatial resolution limitations. Nowadays experimental technologies may enable investigation of magnetic structures near such defects with nanometer resolution.

Experimental observations show that in magnetic films dislocations result in the formation of noncollinear spin structures near dislocation cores: domain walls [5], vortexlike and lobe-shaped magnetic structures [6] that can be referred to chiral couplings induced by elastic torsion.

We develop a micromagnetic model for dislocationinduced DM couplings. As an application, vortex states in thin magnetic film elements are studied under influence of a screw dislocation in their center. This model is relevant for the general problem of chirality selection in magnetic nanostructures [7], [8]. Weak chiral DM couplings reveal themselves only by subtle differences in the magnetization distribution and energetics of non-collinear magnetic states. In magnetic nanodisks the competition between demagnetization energy and exchange couplings causes the formation of vortex states [9], [10]. Such vortices consist

\footnotetext{
a e-mail: g.butenko@ifw-dresden.de
}

of a narrow core with perpendicular magnetization surrounded by an extended area with in-plane magnetization curling around the center (Fig. 1 a) [11], [12]. It has been proposed to use both the up and down polarities, i.e., the perpendicular magnetization of the vortex, or the rotation sense of the curling in-plane magnetization as switchable bit elements in memory devices [13], [14]. In previous work $[15,16]$, we have shown that the homogeneous surfaceinduced DM interactions lift the degeneracy between the four possible vortex states and leads to a chirality selection between the states connected by a global parity change, owing to the broken inversion symmetry at surfaces. This effect will be strongest in ultrathin and clean single-crystalline film elements.

However, a similar chirality selection could be observed also in thicker film elements or even in bulk single crystals under influence of screw dislocation lines. One growth mode of epitaxial films relies on screw dislocations and, alongside the surface-induced symmetry breaking, these defects can change magnetic behavior of such films. In principle, the theoretically anticipated effects could be investigated in experiments on patterned epitaxial film elements.

\section{Equations and methods}

The energy density of a ferromagnet with chiral interactions can be written in the following form:

$$
w=A\left(\partial_{i} m_{j}\right)^{2}-\mathbf{M} \cdot \mathbf{H}-\mathbf{M} \cdot \mathbf{H}_{m} / 2+w_{a}+w_{D},
$$

where $\partial_{i}=\partial / \partial x_{i} ; \mathbf{m}$ is the unit vector along the magnetization $\mathbf{M}=M_{s} \mathbf{m}$ and $M_{s}$ is the saturation magnetization. The exchange couplings are given by the stiffness $A$. $\mathbf{H}$ is the applied magnetic field, and $\mathbf{H}_{m}$ is a self-magnetostatic field. Terms $w_{a}$ and $w_{D}$ represent the anisotropy energy and DM interactions energy correspondingly. 
(a)

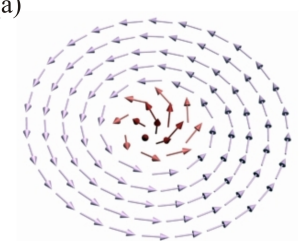

(b)

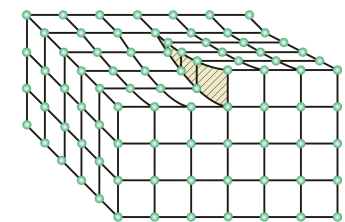

Fig. 1. (a) Vortex state in a circular magnetic nanodisk with axisymmetric magnetization structure. (b) Schematic presentation of a screw dislocation

The anisotropy energy term for the uniaxial ferromagnet is $w_{a}=K_{u}(\mathbf{m} \cdot \mathbf{a})^{2}$, where $\mathbf{a}$ is an anisotropy axis and $K_{u}$ is the anisotropy constant which must be positive in easyplane materials. Within the framework of linear elasticity, considering only lowest-order terms for magnetoelastic (me) interaction, in case of elastic torsions the anisotropy energy density consists of additional term

$$
w_{m e}=C_{i j k l} \epsilon_{i j} \epsilon_{k l} / 2+B_{i j k l} \epsilon_{i j} m_{k} m_{l},
$$

where $C_{i j k l}$ are elastic modules, $B_{i j k l}$ are magnetoelastic coupling constants, $\epsilon_{i j}$ are elastic strains. Strain energy terms caused by magnetization distribution $\mathbf{m}$ and magnetic forces can be neglected for most of the magnetostrictive materials [17]. The strains caused by a dislocation exist independently of the magnetic subsystem and external to it. They are inhomogeneous and lead, particularly, to the local changes in magnetic anisotropy. Outside the core of the defect (the diameter of the dislocation core is about few lattice constants) the strains can be defined in terms of linear elasticity, so the contributions of strains to the total energy density are given by the magnetoelastic energy [17]. In a strained materials this yields an additional contribution for the magnetic anisotropy:

$$
\tilde{w}_{m e}=B_{i j k l} \epsilon_{i j} m_{k} m_{l} .
$$

It may be concluded that defect-initiated strains induce the magnetic anisotropy of the second order of magnetization. In case of a single screw dislocation the only non-zero components of the strain tensor $\epsilon_{i j}$ for an isotropic medium is $\epsilon_{z \varphi}=b /(4 \pi \rho)$ (in presentation of cylindrical coordinates, $b$ is the amplitude of Burgers vector) [18]. Thus, for a uniaxial ferromagnet with anisotropy axis along the dislocation line, defect-induced anisotropy takes the form

$$
\tilde{w}_{a}=K_{i} \frac{m_{z}^{2}}{\rho},
$$

where $K_{i}=B_{z \varphi z z} b / 4 \pi$. From (4) it results that the inhomogeneity region near the dislocation may reach several hundred lattice constants and may exceed the domain wall thickness in the defect-free crystal. Such a drastic change in the local anisotropy may obviously modify the magnetization distribution near a defect.

Broken inversion symmetry can cause local chiral DM interactions, as is the case at surfaces or interfaces of magnetic layers [7], [8]. These antisymmetric DM exchange couplings arise due to spin-orbit effects on the electronic structure due to inversion-asymmetric crystal fields. Within the micromagnetic approach, the DM energy $w_{D}$ induced by the surfaces is described by so-called Lifshitz invariants [2]. In a ferromagnetic system the Lifshitz invariants

are antisymmetric forms linear in first spatial derivatives of the magnetization:

$$
L_{i j}^{(k)}=m_{i} \partial_{k} m_{j}-m_{j} \partial_{k} m_{i},
$$

where $i, j, k$ are certain combination of Cartesian coordinates $x, y, z$. Here, we use an energy density combining Lifshitz invariants (5) for the two in-plane spatial directions,

$$
w_{D}=D_{s}\left(L_{z x}^{(y)}-L_{z y}^{(x)}\right),
$$

which describes the chiral DM exchange that is allowed in symmetries from Laue classes 32, 42, and 62. This $w_{D}$ term favours the curling mode of the magnetization, where the favoured rotation sense is determined by the sign of the material dependent Dzyaloshinskii constant $D_{s}$.

The elastic torsion in the vicinity of a dislocation also breaks inversion symmetry and induces DM couplings. The simplest phenomenological expression for this interaction between torsions and magnetism is given by [19]:

$$
E_{D}=\sum_{\mathbf{R}_{1}, \mathbf{R}_{2}} g\left(\mathbf{R}_{12}\right)\left[\mathbf{S}_{\mathbf{R}_{1}} \times \mathbf{S}_{\mathbf{R}_{2}}\right] \cdot\left(\operatorname{rot} \mathbf{u}_{\mathbf{R}_{1}}-\operatorname{rot} \mathbf{u}_{\mathbf{R}_{2}}\right),(7)
$$

where $\mathbf{u}_{\mathbf{R}}$ is the displacement of the atom at a lattice point $\mathbf{R}$ from its equilibrium position and the energy $g\left(\mathbf{R}_{12}\right)$ determines the strength of the interaction. The induced magnetoelastic coupling vanishes at large distances from the defect core. The defect-induced DM interactions then can be written in a continuum representation for magnetization $\mathbf{M}$ and elastic displacement $\mathbf{u}$ as

$$
w_{D}=D(\mathbf{M} \times \mathcal{D} \mathbf{M}) \cdot \operatorname{rot} \mathcal{D} \mathbf{u},
$$

with $\mathcal{D}=\partial / \partial x+\partial / \partial y+\partial / \partial z$ and $D$ a Dzyaloshinskii constant for the torsional magnetoelastic couplings.

In case of straight screw dislocation there is only one non-zero displacement $u_{z}=b / 2 \pi \cdot \varphi$ with axis $z$ along the dislocation line [18]. Then Eq. (8) takes the form:

$$
\begin{aligned}
w_{D}= & -\frac{D b}{2 \pi} \frac{1}{\rho^{2}}\left[(-\cos 2 \varphi-\sin 2 \varphi)\left(M_{y} \mathcal{D} M_{z}-M_{z} \mathcal{D} M_{y}\right)\right. \\
& \left.+(-\cos 2 \varphi+\sin 2 \varphi)\left(M_{x} \mathcal{D} M_{z}-M_{z} \mathcal{D} M_{x}\right)\right],
\end{aligned}
$$

where $\rho=\sqrt{x^{2}+y^{2}}$.

The equilibrium configurations of $\mathbf{m}$ are derived by minimization of the energy (1) together with the equations of magnetostatics. To describe the vortex states in the circular disk of radius $R_{d}$ and with zero or perpendicular applied field, we consider axisymmetric distributions of the magnetization and express the magnetization vector $\mathbf{m}$ in terms of spherical coordinates and the spatial variables in cylindrical coordinates: $\mathbf{m}=(\sin \theta \cos \psi ; \sin \theta \sin \psi ; \cos \theta)$, $\mathbf{r}=(\rho \cos \varphi ; \rho \sin \varphi ; z)$.

Due to the non-local character of stray-field interactions the micromagnetic problem Eq. (1) constitutes a set of integro-differential equations [10]. In order to simplify this problem we consider the limit of a thin film where the magnetodipole energy has a local character and reduces to a "shape" anisotropy $K_{m}=2 \pi M_{s}^{2}$. This can be added to the uniaxial anisotropy $K_{u}$ yielding a redefinition of the anisotropy energy in Eq. (1) by an effective anisotropy constant $K=K_{u}+2 \pi M_{s}^{2}>0$. We also introduce the characteristic (exchange) length $l_{e}=\sqrt{A / K}$, the anisotropy field 
$H_{a}=2 K / M_{s}$, and a critical value of the Dzyaloshinskii constant $D_{0}=\sqrt{A K}$ as proper material parameters of the problem. The field $H_{a}$ determines the equilibrium magnetization of homogeneously magnetized defect-free layers in an applied perpendicular field $H, \cos \theta_{h}=H / H_{a}$. For $|D| / D_{0}>4 / \pi=1.273$ the magnetization of a layer transforms into a modulated state $[2,20]$. The exchange length $l_{e}$ gives a characteristic radius of the vortex core. Most experimentally investigated nanodisks have radii much larger than the exchange length, $R_{d} \gg l_{e}$. In this case vortices consist of a strongly localized core encircled by a wide ring with a constant polar angle $\theta=\theta_{h}$ (Fig. 1 a).

The variational problem for functional (1) has the rotationally symmetric solution $\psi=\varphi \pm \pi / 2, \theta=\theta(\rho)$. By substituting the solutions for $\psi$ into Eq. (1) and integrating with respect to $\varphi$ the vortex energy can be reduced to the following form $E=2 \pi \int_{0}^{R_{d}} w^{(d)}(\rho) \rho d \rho$ with

$$
\begin{aligned}
w^{(d)}(\rho)= & A\left[\left(\frac{d \theta}{d \rho}\right)^{2}+\frac{\sin ^{2} \theta}{\rho^{2}}\right]+K \cos ^{2} \theta-H M_{s} \cos \theta \\
& -D_{s}\left(\frac{d \theta}{d \rho}+\frac{\cos \theta \sin \theta}{\rho}\right) \\
& -\frac{D_{d} l_{e}^{2}}{\rho^{2}}\left(\frac{d \theta}{d \rho}-\frac{\sin \theta \cos \theta}{\rho}\right)+\frac{K_{d} l_{e}}{\rho} \cos ^{2} \theta
\end{aligned}
$$

where the dislocation coincides with the disk center and the magnetic field $H$ is assumed to be perpendicular to the disk plane. For the dislocation-induced couplings, we introduce corresponding coefficients $D_{d}=D M_{S}^{2} / l_{e}$ for the chiral interactions and $K_{d}=K_{i} / l_{e}$ for the magnetic anisotropy.

The variational problem for functional (10) reduces to an Euler equation for $\theta(\rho)$ :

$$
\begin{aligned}
& A\left(\frac{d^{2} \theta}{d \rho^{2}}+\frac{1}{\rho} \frac{d \theta}{d \rho}-\frac{1}{\rho^{2}} \sin \theta \cos \theta\right)+K \sin \theta \cos \theta-H M_{s} \frac{\sin \theta}{2} \\
& -\frac{D_{s}}{\rho} \sin ^{2} \theta-\frac{2 K_{d} l_{e}}{\rho} \sin \theta \cos \theta-\frac{D_{d} l_{e}^{2}}{\rho^{3}} \cos 2 \theta=0 .
\end{aligned}
$$

With the boundary conditions

$$
\theta(\delta)=0, \quad(d \theta / d \rho)_{\rho=R_{d}}=g\left(\theta, R_{d}\right)
$$

the solutions of Eq. (11) yield the equilibrium vortex profiles. Here, $\delta$ is a dislocation core size where the linear elasticity theory is not applicable and where the magnetoelastic couplings must be considered separately. The size of this core region is of the order of the Burgers vector modulus $b$. Reasonable values for $\delta$ are in the range $b$ to $4 b$, i.e. $\delta \lesssim 1 \mathrm{~nm}$ in most cases. In this area induced local magnetic anisotropy likely predominates other interactions and we assume a homogeneously magnetized solution $\theta(\rho<\delta) \equiv 0$. In the limit of $\rho \rightarrow R_{d}$ with $R_{d} \gg l_{e}$ the energy of the vortex in a nanodisk with a dislocation $w^{(d)}$ (10) converges towards the value of energy for vortices in a defect-free nanodisk [15], [16]. Correspondingly, for the finite disk, free boundary conditions $g\left(\theta, R_{d}\right)=0$ can be used. The procedure for deriving the solutions of boundary problem (11) and (12) is the same as for dislocation-free nanodisks described in Refs. [15], [16].

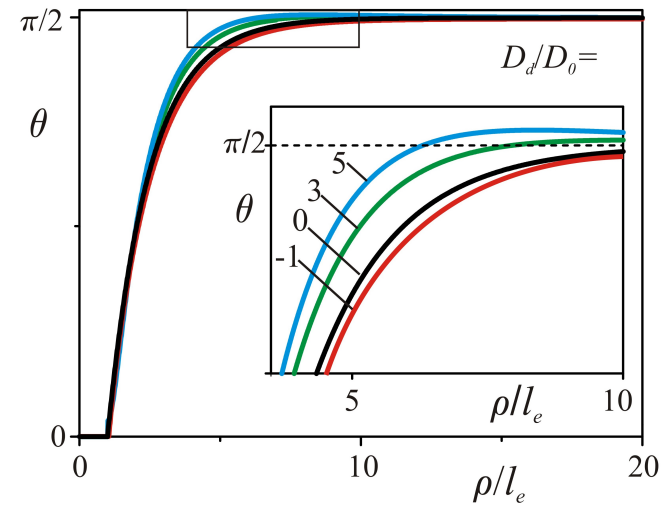

Fig. 2. Typical dependence of solutions $\theta(\rho)$ on dislocationinduced DM interactions in zero applied field in absence of intrinsic and surface-induced DM couplings with $K_{i} / K_{0}=0.1$. The vortices slightly widen with $D_{d}<0$ and shrink for $D_{d}>0$. Inset magnifies the region marked by a box.

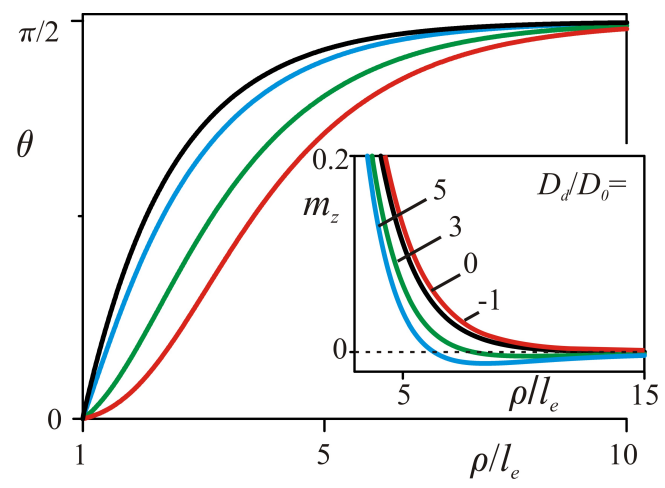

Fig. 3. Typical dependence of solutions $\theta(\rho)$ on dislocationinduced easy axis anisotropy in zero applied field without intrinsic and surface-induced DM couplings with $D_{d} / D_{0}=-0.5$. The corresponding values of $K_{i} / K$ (from top to bottom): $0,0.8$, 1.6, 2.0. Anisotropy extends the region with near-perpendicular magnetization. Inset shows the magnetization profiles $m_{z}(\rho)$ for different values of $D_{d}$ in zero applied field in absence of intrinsic and surface-induced DM couplings with $K_{i} / K_{0}=0.1$. Positive $D_{d}$ decreases the central spot with positive $m_{z}$ and widens the ring with negative.

\section{Vortex solutions and Magnetization profiles}

Here, representative results on the effect of a dislocation on the vortex structure in a magnetic nanodisk are reported for systems with fixed disk radius $R_{d}=30 l_{e}$ and a dislocation core size $\delta=l_{e}$. These solutions well represent the vortexcore properties for any $R_{d} \gg l_{e}$. As shown previously, homogeneous surface-induced DM couplings favour vortices with the same chirality [15], [16] extending their core sizes for positive $D_{s}$. For negative $D_{s}$, the vortex structure changes its character: the vortex core consists of a narrower internal and an adjacent ring with a reverse magnetization rotation. The effects of the inhomogeneous dislocationinduced DM couplings are similar, Fig. 2. In case of negative $D_{d}$ the angles $\theta(\rho)$ vary monotonically from zero at the dislocation core to $\theta_{h}$. In this case the local chirality of magnetization is everywhere favoured by the induced DM interactions and the core size widens with increasing $\left|D_{d}\right|$. When $D_{d}$ becomes positive the magnetization in the core has unfavourable chirality and after a maximal value $\theta$ the 


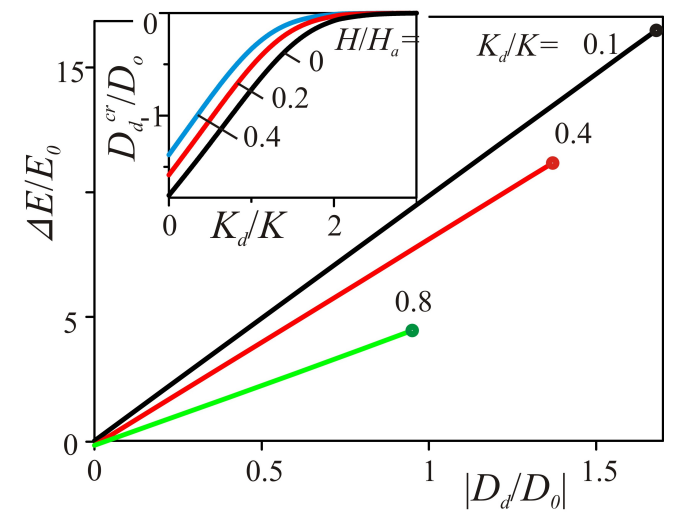

Fig. 4. Energy difference for vortices with opposite chirality as a function of dislocation-induced DM interactions for different values of induced anisotropy $\left(E_{0}=D_{0} A / K\right)$. Inset present critical value of unfavourable $D_{d}$ that destroys the static vortex states.

slope $\theta_{\rho}$ becomes negative, and the magnetization structure changes its local chirality. After that the polar angle $\theta(\rho)$ monotonically approaches the limiting value $\theta_{h}$.

Induced uniaxial anisotropy predictably also widens the vortex core and extends the region with perpendicular magnetization (Fig. 3), thereby increasing total magnetization of the disk. Strong enough induced anisotropy destroys the vortex structure and the homogeneously magnetized state with perpendicular magnetization occurs.

The character of the vortex profiles for different signs of $D_{d}$ are reflected in their magnetization distribution (Fig. 3 Inset). Negative $D_{d}$ increases the width of the central spot with $m_{z}>0$. As a result the total perpendicular magnetization of the disk $\left\langle m_{z}\right\rangle$ is larger in vortices of the right positive chirality. Positive $D_{d}$ squeezes the central magnetization core with $m_{z}>0$ and widens the adjacent ring with negative perpendicular magnetization $\left(m_{z}<0\right)$.

In Fig. 4 the energy difference $\Delta E=E_{p}-E_{n}$ between vortices with different chirality is shown for the models combining dislocation-induced DM couplings and uniaxial anisotropy. The results show that the dislocation-induced DM couplings violate chiral symmetry and select vortices with one sign of the global chirality as energetic groundstate, as in the case of surface-induced chirality selection. If the dislocation-induced DM interactions become very strong, they also restrict the existence range of the vortex state: for some critical value of negative $D_{c r}$ the chiral energy contributions outweighs others energies and stabilizes twisted magnetization structures (e.g. vortices with oscillations on the outskirt). These states are not solutions of the geometrically restricted variational problem with free boundary conditions (11)-(12). The dependence of the critical induced DM-couplings on induced anisotropy and applied fields are depicted in Fig. 4. Because the induced anisotropy also destroys the vortex states, the magnitude of $D_{c r}$ is reduced with increasing $K_{d}$ (Inset Fig. 4).

\section{Conclusions}

We have introduced a continuum description of chiral inhomogeneous Dzyaloshinskii-Moriya interactions, that are induced by torsional strains (Eq.(8)). This is a general formulation that is applicable in micromagnetic models of ferromagnets with such symmetry-lowering effects. Possible effects of these couplings will be particularly strong owing to the large strains near defects. This has been illustrated by a micromagnetic model for a disklike film element with a screw dislocation at its center, where the effect leads to a chirality selection on the vortex state. Similar effects related to the strong DM-couplings near dislocations also could be responsible for the chirality selection on noncollinear magnetization structures in distorted bulk crystals, as observed for the handed helix domain populations in Ho-single crystals under torsion [19].

\section{References}

1. A. Arrott, J. Appl. Phys. 34, (1963) 1108.

2. I. E. Dzyaloshinskii, Sov. Phys. JETP 19, (1964) 960.

3. H. Kronmüller and M. Fähnle, Micromagnetism and the Microstructure of ferromagnetic solids (Cambridge University Press, 2003).

4. H. Träuble, The influence of crystal defects on magnetization processes in ferromagnetic single crystals in Magnetism and Metallurgy (Academic Press, San Diego, 1969).

5. R. Ravlíc, M. Bode, A. Kubetzka, and R. Wiesendanger, Phys. Rev. B 67, (2003) 174411.

6. L. Berbil-Bautista, S. Krause, M. Bode, A. BadíaMajós, C. de la Fuente, R. Wiesendanger, and J.I. Arnaudas, Phys. Rev. B 80, (2009) 241408.

7. A. Crépieux and C. Lacroix, J. Magn. Magn. Mat. 182, (1998) 341.

8. A.N. Bogdanov and U.K. Rößler, Phys. Rev. Lett. 87, (2001) 037203.

9. E. Feldtkeller and H. Thomas, Phys. Kondens. Mater. 4, (1965) 8.

10. A. Hubert and R. Schäfer, Magnetic Domains: The Analysis of Magnetic microstructure (Springer, Berlin, 1998).

11. T. Shinjo, T. Okuno, R. Hassdorf, K. Shigeto, and T. Ono, Science 289, (2000) 930.

12. A. Wachowiak, J. Wiebe, M. Bode, O. Pietzsch, M. Morgenstern and R. Wiesedanger, Science 298, (2002) 577.

13. K. Bussmann, G.A. Prinz, S.-F. Cheng, and D. Wang, Appl. Phys. Lett. 75, (1999) 2476.

14. S. Bohlens, B. Krüger, A. Drews, M. Bolte, G. Meier, and D. Pfannkuche, Appl. Phys. Lett. 93, (2008) 142508.

15. A.B. Butenko, A.A. Leonov, A.N. Bogdanov, and U.K. Rößler, Phys. Rev. B 80, (2009) 134410.

16. A.B. Butenko, A.A. Leonov, A.N. Bogdanov, and U.K. Rößler, J. Phys.: Conf. Ser., 200, (2010) 042012.

17. A.B. Dichenko and V.V. Nikolayev, J. Magn. Magn. Magn. 53, (1985) 71.

18. L.D. Landau and E.M. Lifschitz, Theory of elasticity (Pergamon press, 1975)

19. V.I. Fedorov, A.G. Gukasov, V. Kozlov, S.V. Maleyev, V.P. Plakhty, and I.A. Zobkalo, Phys. Lett. A 224, (1997) 372-378.

20. A. Bogdanov and A. Hubert, J. Magn. Magn. Mater. 138, (1994) 255. 